\title{
SIVmac239 MVA Vaccine with and without a DNA Prime, Similar Prevention of Infection by a Repeated Dose SIVsmE660 Challenge Despite Different Immune Responses
}

\author{
Lilin Lai ${ }^{\mathrm{a}}$, Sue-Fen Kwa ${ }^{\mathrm{a}}$, Pamela A. Kozlowski ${ }^{\mathrm{b}}$, David C. Montefiori ${ }^{\mathrm{c}}$, Tracy Nolen ${ }^{\mathrm{d}}$, \\ Michael G. Hudgens ${ }^{d}$, Welkin E. Johnson ${ }^{e}$, Guido Ferrari ${ }^{\mathrm{c}}$, Vanessa M. Hirsch ${ }^{\mathrm{h}}$, Barbara K. \\ Felber $^{f}$, George N. Pavlakis ${ }^{g}$, Patricia L. Earl ${ }^{\mathrm{h}}$, Bernard Moss ${ }^{\mathrm{h}}$, Rama Rao Amara ${ }^{\mathrm{a}, \mathrm{i}}$, and \\ Harriet L. Robinson ${ }^{j}$ \\ ${ }^{a}$ Division of Microbiology and Immunology, Yerkes National Primate Research Center, Emory \\ University, Atlanta, Georgia 30329 \\ bDepartment of Microbiology, Immunology, and Parasitology, Louisiana State University Health \\ Sciences Center, New Orleans, Louisiana 70112 \\ 'Laboratory for AIDS Vaccine Research and Development, Department of Surgery, Duke \\ University Medical Center, Durham, North Carolina 27710 \\ dDepartment of Biostatistics, University of North Carolina, Chapel Hill, North Carolina 27599 \\ eNew England National Primate Research Center, Harvard University, Southborough, \\ Massachusetts 01772 \\ fHuman Retrovirus Pathogenesis Section, Vaccine Branch, National Cancer Institute, Frederick, \\ Maryland 21702 \\ gHuman Retrovirus Section, Vaccine Branch, National Cancer Institute, Frederick, Maryland \\ 21702 \\ hLaboratory of Viral Diseases, National Institute of Allergy and Infectious Diseases, National \\ Institutes of Health, Bethesda, Maryland 20892 \\ a,i Vaccine Research Center and Department of Microbiology and Immunology, Emory University, \\ Atlanta, Georgia 30329 \\ iGeoVax, Inc., Smyrna, Georgia 30080
}

\begin{abstract}
Background-Vaccine regimens using different agents for priming and boosting have become popular for enhancing $\mathrm{T}$ cell and $\mathrm{Ab}$ responses elicited by candidate HIV/AIDS vaccines. Here we use a simian model to evaluate immunogenicity and protective efficacy of a recombinant modified vaccinia Ankara (MVA) vaccine in the presence and absence of a recombinant DNA prime. The simian vaccines and regimens represent prototypes for candidate HIV vaccines currently undergoing clinical testing.
\end{abstract}

\footnotetext{
(C) 2011 Elsevier Ltd. All rights reserved.

Publisher's Disclaimer: This is a PDF file of an unedited manuscript that has been accepted for publication. As a service to our customers we are providing this early version of the manuscript. The manuscript will undergo copyediting, typesetting, and review of the resulting proof before it is published in its final citable form. Please note that during the production process errors may be discovered which could affect the content, and all legal disclaimers that apply to the journal pertain.
} 
Method-Recombinant DNA and MVA immunogens expressed simian immunodeficiency virus (SIV)mac239 Gag, PR, RT, and Env sequences. Vaccine schedules tested inoculations of MVA at months 0,2 , and 6 (MMM regimen) or priming with DNA at months 0 and 2 and boosting with MVA at months 4 and 6 (DDMM regimen). Twelve weekly rectal challenges with the heterologous SIV smE660 were initiated at 6 months following the last immunization.

Results-Both regimens elicited similar 61-64\% reductions in the per challenge risk of SIVsmE660 transmission despite raising different patterns of immune responses. The DDMM regimen elicited higher magnitudes of CD4 T cells whereas the MMM regimen elicited higher titers and greater avidity Env-specific IgG and more frequent and higher titer SIV-specific IgA in rectal secretions. Both regimens elicited similar magnitudes of CD8 T cells. Magnitudes of T cell responses, specific activities of rectal $\operatorname{IgA~Ab}$, and the tested specificities for neutralization and antibody-dependent cellular cytotoxicity did not correlate with risk of infection. However, the avidity of Env-specific IgG had a strong correlation with the per challenge risk of acquisition, but only for the DDMM group.

Conclusions-We conclude that for the tested immunogens in rhesus macaques, the simpler MMM regimen is as protective as the more complex DDMM regimen.

\section{Keywords}

Vaccine; Immunodeficiency virus; Simian immunodeficiency virus; DNA vaccine MVA vaccine; avidity in protection

\section{Introduction}

In the past 20 years, heterologous prime-boost vaccination regimens have become a popular method for enhancing the elicitation of $\mathrm{Ab}$ and $\mathrm{T}$ cell responses by recombinant DNA, viralvectored and protein vaccines[1,2]. In a heterologous regimen, one agent is used to prime the immune response, and a second, to boost the immune response. The priming and boosting agents share immunogens but are otherwise immunologically distinct. Immune responses elicited by the 1st agent focus and augment the immune response on the immunogen common to both agents. When used together, agents that elicit low titer immune responses in homologous priming and boosting regimens, achieve higher titer responses. The technique has been particularly popular for eliciting $\mathrm{T}$ cell responses [3, 4], but can also be effective at enhancing Ab responses[5] [6].

In 2002, using SHIV-89.6 immunogens, we reported that MVA immunizations in the presence or absence of a DNA prime elicited different patterns of immune responses but similar patterns of protection for a high dose intrarectal SHIV-89.6P challenge[7]. Here, we use a SIVmac239 (SIV239) MVA vaccine in the presence and absence of a SIV239 DNA prime and again find similar patterns of protection; this time for a repeated SIVsmE660 (SIVE660) rectal challenge, despite the elicitation of different patterns of immune responses [8-10]. Our results again show the DNA prime enhancing T cell responses and the MVAonly immunizations eliciting higher $\mathrm{Ab}$ responses. Interestingly, the higher Env-specific $\mathrm{Ab}$ responses in the MVA-only group did not translate into better prevention of acquisition. We discuss factors that could have caused differences in the protective activity of Ab elicited by the DDMM and MMM regimens.

\section{Materials and Methods}

\subsection{Vaccines}

DNA vaccine plasmid Rama37 expresses SIV239 Gag, PR, RT, Env, Tat, and Rev from a single RNA by subgenomic splicing and frameshifting. A single recombinant MVA 
designated DR1 or MVASIVgpe expressed SIV239 Gag, PR, RT and Env [11] [12]. The MVA vaccine expressed virus like particles whereas the over-expressed Gag in the DNA vaccine formed intracellular aggregates as well as virus like particles. The DNA vaccine expressed the complete gp160 form of Env and the MVA vaccine encoded a gp150 form of Env that had been truncated for 146 amino acids at the C-terminus of its gp41 subunit to enhance expression on the plasma membrane of infected cells and stabilize the insert[13].

\subsection{Study Design}

Animal studies were conducted at the Yerkes National Primate Research Center and approved by the Emory University Animal Care and Use Committee. Young adult rhesus macaques of Indian origin, pre-screened to preclude the presence of the Mamu-A*01 and limit the presence of the Maтu- $B^{*} 08$ and $B^{*} 17$ histocompatibility types to no more than one per group [9], were randomized into groups of 8 each. Immunization regimens were the same as those being used in human trials[14]. For DNA/MVA immunizations, $3 \mathrm{mg}$ of the DNA vaccine (D) was administered at weeks 0 and 8 and $1 \times 10^{8}$ plaque forming units of the MVA (M) vaccine at weeks 16 and 24 (DDMM regimen). For MVA priming and boosting, $1 \times 10^{8}$ plaque forming units of the MVA vaccine was administered at weeks 0,8 and 24 (MMM regimen). All vaccinations were delivered intramuscularly by hypodermic needle and syringe. Twelve weekly intrarectal challenges were initiated 6 months after the final MVA immunization using 5000 tissue culture infectious doses $_{50}\left(1.8 \times 10^{7}\right.$ copies of viral RNA) of the SIVE660-Hirsch2001 stock as previously described[15]. Unvaccinated controls included nine 2-4 year old male Indian rhesus macaques that were negative for $М а т и A * 01$, $B^{*} 08$ and $B^{*} 17$, which underwent simultaneous challenge with the two vaccine groups; six 2-4 year old Indian rhesus males positive for Мати $A * 01$ that were challenged in an overlapping trial at the Yerkes Primate Research Center, and eight 10-13 year old Indian rhesus with mixed $A * 01$ and $B * 17$ Mamu types challenged at the National Cancer Institute under the same conditions and with the same stock. TRIM $5 \alpha$ genotype was determined by sequence analysis of PCR fragments [16].

\subsection{Humoral immune assays}

Titers of Env-specific IgG and avidity indices, or the fraction of bound $\operatorname{IgG}$ resisting a $1.5 \mathrm{M}$ NaSCN wash $\times 100$, were determined using ELISAs with SIV239 Env captured from VLPs and SIVE660 ENV captured from virions as antigens [17]. Concentrations of Env-specific; Gag, Pol-specific and total IgA in rectal secretions [18]were determined by ELISA using plates coated respectively with rgp130mac251 (Immunodiagnostics, Woburn, MA), SIV lysate (Advanced Biotechnologies Inc., Columbia, MD) and goat anti-monkey IgA (AlphaDiagnostics, San Antonio, TX) [15, 19]. Neutralization assays were conducted using pseudovirions or infectious viruses and a luciferase reporter gene assay in TZM-bl cells or infections virus and M7-luc cells[20]. Assays for antibody dependent cellular cytotoxicity (ADCC) were conducted using SIVmac239 gp120 (Immune Tech Corp) to coat CEM.NKR ${ }_{\text {CCR5 }}$ cells[21].

\subsection{Cellular immune assays}

Cellular immune assays and breadth of responses were conducted using stimulations of PBMCs with pools of peptides (15 mers overlapping by 11) matched to the SIV239 immunogen [17]. Responding cells were scored using intracellular cytokine staining (ICS) [22]. Breadth of responses was tested using $13 \mathrm{Gag}$ and 11 Env peptide pools. Boolean analysis was performed to measure polyfunctionality[23]. 


\section{$1.5 \mathrm{~T}$ cells in rectal biopsies}

Approximately 15-20 colorectal pinch biopsies were digested with $200 \mathrm{U} / \mathrm{ml}$ of collagenase IV (Worthington; Lakewood, NJ) with agitation for $2 \mathrm{hr}$ at $37^{\circ} \mathrm{C}$. Tissue pieces were further dissociated using a syringe with 18- and then 16-gauge blunt-end needles. The resultant cell suspension was filtered with a cell strainer and used for flow cytometric analysis.

\subsection{Statistics}

Comparisons of immune responses between groups were done using the Wilcoxon test (comparisons between 2 groups) and Kruksal-Wallis test (comparisons between 3 groups). Correlations between immune responses and the number of challenges to infection used the Spearman test. Comparisons of number of challenges until infection between groups were done using exact log-rank tests. Models of the protective effect of vaccination were fit to data as previously described and Akaike Information Criteria (AIC) used to select the most parsimonious model[24, 25]. All tests were two tailed with $\mathrm{p}<0.05$ considered significant. Analyses were not corrected for multiple comparisons.

\section{Results}

\subsection{Vaccine trial and elicited $T$ cell responses}

Groups of 8 rhesus were immunized with two inoculations of the SIV239 DNA vaccine at weeks 0 and 8 followed by two inoculations with the SIV239 MVA vaccine at weeks 16 and 24 (DDMM regimen) or three MVA inoculations at weeks 0, 8 and 24 (MMM regimen). In the DDMM group, $T$ cell responses were not detected until after the $1^{\text {st }}$ MVA inoculation, at which time both CD4 and CD8 T cells achieved their maximal magnitudes with medians of $1.5 \%$ of total CD4 T cells and $0.09 \%$ of total CD8 T cells (Fig. 1, A and C). For the MMM regimen, both $\mathrm{CD} 4$ and $\mathrm{CD} 8 \mathrm{~T}$ cells were near maximal magnitudes following the $1^{\text {st }} \mathrm{MVA}$ immunization and peaked with medians of $0.19 \%$ of total CD4 T cells and $0.13 \%$ of total CD8 $\mathrm{T}$ cells following the $2^{\text {nd }}$ MVA immunization (Fig. 1, B and D). The MMM regimen elicited the highest CD4 response early in the regimen ( 2 and 9 weeks in the trial), whereas the DDMM regimen elicited the highest CD4 response late in the regimen (17, 25 and 37 weeks in the trial) (Fig. 1A and B). In contrast, the MMM regimen raised the highest CD8 responses both early and late in the trial (2,9 and 37 weeks), while both regimens had similar magnitudes of responses following the final MVA boost (week 25) (Fig. 1C and D).

The highest magnitude CD4 and CD8 responses also had the greatest breadth (Fig. 2A and B). Following the $1^{\text {st }}$ MVA boost In the DDMM regimen, when elicited CD4 cells were much higher than elicited CD8 T cells, CD4 responses had a median breadth of 9.5 pools and CD8 responses a median breadth of 1.5 pools. These breadths increased to medians of 12 pools for the CD4 response and 4.5 pools for the CD8 response following the $2^{\text {nd }}$ MVA boost. In contrast, for the MMM regimen, where magnitudes of responses were overall similar, CD4 responses had median breadths of 4.5 and 3 pools respectively after the $1^{\text {st }}$ and $2^{\text {nd }}$ MVA immunizations; and CD8 responses, median breadths of 4 pools following both the $2^{\text {nd }}$ and $3^{\text {rd }}$ MVA boosts. For both regimens, the $\mathrm{T}$ cell responses were balanced between Gag and Env (Fig. 2, C and D).

Responding cells were analyzed for patterns of co-production of IFN- $\gamma$, IL-2 and TNF- $\alpha$ (Fig. 3). The DNA primed-CD4 responses showed higher percentages of IFN- $\gamma$, IL-2 and TNF- $\alpha$ triple producers than the MMM regimen (55\% as opposed to 26\%) whereas the MMM elicited responses showed higher percentages of IFN- $\gamma$ and TNF- $\alpha$ double producers (27\% as opposed to $16 \%$ ), and IFN- $\gamma$ single producing cells (20\% as opposed to $6 \%$ ) than the DDMM regimen (Fig. 3A). For both regimens, the most frequent pattern of cytokine coproduction for CD8 T cells was co-production of IFN- $\gamma$ and TNF- $\alpha$ : $43 \%$ of the DDMM- 
elicited CD8 and 63\% of the MMM-elicited CD8 cells coproduced IFN- $\gamma$ and TNF- $\alpha$ (Fig. 3C). Despite this similarity, the functionality of the CD8 cells elicited by the 2 regimens differed in 4 out of 7 tested patterns of cytokine co-production.

\subsection{Vaccine-elicited antibody responses}

The MVA-only immunizations raised by far the stronger antibody responses (Fig. 1E-F). In contrast to $\mathrm{T}$ cell responses that reached their highest levels in blood early (MMM) or midway (DDMM) through immunization regimens, the titers of Env-specific IgG increased with each boost. Following the last immunization, the MMM regimen had elicited a median titer of $14 \mu \mathrm{g}$ per ml; and the DDMM regimen, a median titer of $6.6 \mu \mathrm{g}$ per $\mathrm{ml}$ of Ab specific for the SIV239 Env of the immunogen (Fig. 4A). Titers of binding Ab for the E660 Env of the challenge virus were about 2-fold lower(Fig. 4A).

The MMM immunizations also elicited much higher avidity Env-specific IgG than the DDMM immunizations (Fig. 4B). The avidity index for the MMM immunizations increased from a mean of 40 post the $2^{\text {nd }}$ MVA boost to a mean of 55 post the $3^{\text {rd }}$ MVA boost. Interestingly, the avidity indices for the immunizing and challenge Env were similar for the MMM-elicited Env-specific IgG (indices of 54) whereas, for the DDMM-elicited Ab, they were higher for the challenge Env (index of 36) than for the immunizing Env (index of 29) $(\mathrm{p}=0.0008)$.

Despite the differences in titers and avidity of elicited Env-specific IgG, the DDMM and MMM immunizations elicited overall similar titers of neutralizing Ab (Fig. 4C). Both regimens elicited neutralizing $\mathrm{Ab}$ for tier 1 (easy to neutralize) viruses (Fig. 4C) whereas neither raised detectable responses for tier 2 (harder to neutralize) viruses (data not shown) [26]. A HIV-2 virus (7312A/V33M) developed to test for the presence of neutralizing Ab for the CD4i site in the presence of sub-inhibitory doses of soluble CD4 scored for similar low activities in the presence and absence of sCD4 indicating that antibodies to the CD4i site had not been induced[27]. The SIVE660 stock grown on CEMx174 cells, which exhibits tier 2 characteristics, did not score in the TZM-bl assay but did score at low levels when assayed on M7-luc cells (data not shown).

ADCC activity was measured for sera harvested at peak responses and pre-challenge. This activity was significantly higher for pre-challenge sera from the MMM group (Fig. 4D).

The MMM regimen also elicited higher IgA responses in rectal secretions than the DDMM regimen (Fig. 4E and F). Env-specific IgA was detected in 5 of $8 \mathrm{MMM}$ vaccinated animals but in only 1 of 8 DDMM vaccinated animals; Gag,Pol specific IgA scored in 7 of 8 MMM vaccinated animals but none of the DDMM group. The IgA responses persisted within 2fold of their peak in memory, whereas the median levels of SIV-specific IgG had declined by about 5 -fold by week 37 .

\subsection{Repeat dose rectal challenge}

A repeat dose rectal challenge with 5000 TCID $_{50}$ of the heterologous SIVE660 was initiated at 6 months after the last MVA immunization. To evaluate the consistency and potential effects of Мати type and age on the challenge, we compared the results from 3 independent challenge studies in Indian rhesus of different ages and Мати type conducted at two different institutions under the same conditions with the same SIVE660 stock. The data from these three studies showed no differences in acquisition curves (Fig. 5A). To increase the power of the current study we have combined data from the 3 control groups for a total group size of 23 . 
Kaplan-Meier curves for the prevention of acquisition were similar for the DDMM and MMM regimens (Fig. 5B). Whereas 22 out of 23 of the unvaccinated animals were infected by the $6^{\text {th }}$ challenge, only 3 out of 8 MMM animals and 5 out of 8 DDMM vaccinated animals had acquired virus following the first 6 challenges. By the $12^{\text {th }}$ challenge, the one remaining unvaccinated animal was infected whereas in both vaccine groups two out of 8 animals remained uninfected. The exact log-rank test indicates a significant difference between the control and DDMM group ( $\mathrm{p}=0.01$ ), and between the control and the MMM group ( $\mathrm{p}=0.004)$, but not between the DDMM and MMM groups ( $\mathrm{p}>0.05)$.

In modeling the protective effect for both regimens, the smallest AIC values were achieved by assuming the vaccines had a leaky effect and there was no heterogeneity between animals in susceptibility. For this model, the estimated probability of infection per challenge was 0.31 for an unvaccinated animal and 0.12 for DDMM vaccinated animals and 0.11 for MMM vaccinated animals corresponding to $61 \%$ (95\% CI 0.15.-0.83) and 64\% (95\% CI $0.14-0.76$ ) reductions of the per challenge infection risk due to DDMM or MMM vaccination respectively.

Analyses for levels of post challenge virus and loss of rectal CD4 T cells revealed no significant differences between the vaccine groups and the unvaccinated group (Fig. $5 \mathrm{C}$ and D). For these analyses, only the 9 animals in the simultaneously challenged group were used as unvaccinated controls because post infection data is subject to Mamu-restriction[28, 29].

\subsection{Post infection immune responses}

By two weeks post infection, the vaccinated and unvaccinated animals had similar magnitudes of SIV-specific T cell responses but highly dissimilar Env-specific Ab responses (Fig. 6). Env-specific IgG and IgA responses were 50-fold higher in the vaccinated groups and remained higher for the next 6 months. Despite the difference in pre-challenge IgA responses, the two vaccine regimens had similar tempos and heights for their anamnestic $\operatorname{Ig} \mathrm{A}$ responses.

Although the post infection $\mathrm{T}$ cell response did not show differences in magnitudes, they did show differences in functionality (Fig. 3B \& D). For CD8 T cells, some of these differences were the same as those seen post vaccination.

\subsection{Correlations}

In the DDMM group, the number of exposures until infection correlated directly with the avidity of the Env-specific IgG response for the E660 Env of the challenge virus ( $\mathrm{r}=0.9$, p $<0.0001)(F i g .7 A)[15]$. This same correlation was not seen for the higher avidity Envspecific IgG elicited by the MMM vaccinations (Fig. 7B). Risk for infection for the DDMM group correlated with the avidity of the response for the E660 Env of the challenge virus, but not with the avidity for the 239 Env of the immunogen (data not shown). No correlations were found between the number of challenges to infection and neutralizing activity (at peak response), ADCC (either at peak response or prechallenge), the presence of rectal IgA (at peak response), and the magnitudes and breadths of total, Gag, or Env T cell responses (post the $1^{\text {st }}$ and $2^{\text {nd }}$ MVA boosts for the DDMM regimen and post the $2^{\text {nd }}$ and $3^{\text {rd }}$ MVA inoculations for the MMM regimen). Data for individual animals can be seen in supplementary Table 1. Genotyping of the study macaques for TRIM5 $\alpha$ alleles suggested that Trim5 had not played a role in prevention of infection (Fig. 7C) [16].

\subsection{Discussion}

The SIV239 DDMM and MMM vaccine regimens raised similar levels of protection against a repeated SIVE660 rectal challenge despite different patterns of immune responses. 
Analyses for transmission risk were most consistent with a leaky vaccine effect in which the DDMM group had a 61\% lower chance and the MMM group a 64\% lower chance of infection at each challenge than the unvaccinated group.

For the DDMM regimen, the avidity of vaccine-elicited IgG for the Env of the SIVE660 challenge was a strong correlate for reduced risk of infection. Acquisition did not occur in the two animals with avidity indices of $>40$. Thus, an approach to increasing protection in the DDMM regimen would be to increase the avidity of the Env-specific IgG by an additional MVA boost $[17,30]$. Increased avidity can also be achieved by priming with a DNA vaccine that co-expresses GM-CSF with viral proteins [15]. The association between avidity and protection presumably reflects $\mathrm{Ab}$ functions, which depend on the affinity of $\mathrm{Ab}$ binding. Such functions include the breadth and strength of neutralizing activity [31] as well as Fc-mediated activities such as ADCC, the activation of complement-mediated lysis, opsonization and phagocytosis, and antibody-dependent virus trapping [32-34].

In contrast to the DDMM regimen, the MMM regimen did not elicit Ab with protective avidity. In the MMM group, all of the animals had avidity indices above the protective index for the DDMM group, yet only two remained uninfected. We suggest that this reflects differences in conformational and linear epitopes displayed by the full length gp160 Env expressed by the DNA vaccine and the C-terminal truncated gp150 Env expressed by the MVA[13]. C-terminal truncations of Env affect structure as well as function and can result in Envs with greater exposure of the CD4 binding site[35] [36], increased fusion potential and altered Env incorporation and stability in virions[37-40]. Priming with a gp160 Env in the DDMM regimen, as opposed to priming with a gp150 Env in the MMM regimen has the potential for biasing immune responses for different $\mathrm{Ab}$ specificities by virtue of the priming immunization establishing antigenic $\sin [41,42]$. Indeed, a difference in the specificity of the $\mathrm{Ab}$ elicited by DDMM and MMM regimens is suggested by these two regimens eliciting overall similar titers of neutralizing Ab despite the MMM regimen eliciting 5-times higher titers and much higher avidity Env-specific Ab. The Ab elicited by these two regimens also had different relative avidities for the 239 Env of the immunogen and the E660 Env of the challenge virus. If the specificity of the Ab elicited by the DDMM and MMM regimens had been similar, increased titers of higher avidity Ab should have resulted in increased titers of neutralizing $\mathrm{Ab}[15]$ and relative avidities for the two test Envs should have been the same.

Thus, we suggest that not all high avidity $\mathrm{Ab}$ is protective $\mathrm{Ab}$ and that for high avidity $\mathrm{Ab}$ to be protective, it must target conserved conformational and linear epitopes for the Env on the challenge virus. Indeed, the avidity of the DDMM-elicited Ab for the E660 Env of the challenge virus correlated with the number of challenges to infection whereas the avidity of this same $\mathrm{Ab}$ for the 239Env of the immunogen did not show the correlation. For the avidity to be protective, it had to represent $\mathrm{Ab}$ that recognized the challenge virus.

Important goals for future studies are to test a full length gp160 Env in MVA for its ability to elicit protective avidity for a heterologous challenge. Given the good ability of the MMM regimen to elicit $\mathrm{Ab}$, the potential of this regimen to elicit $\mathrm{Ab}$ with protective avidity warrants further development. If such can be achieved, our recombinant MVA vaccine, which has proven effective at eliciting Env-specific Ab in humans[14], could provide a single modality HIV/AIDS vaccine with an Ab correlate for protection.

\section{Supplementary Material}

Refer to Web version on PubMed Central for supplementary material. 


\section{Acknowledgments}

We thank Dr. Francois Villinger and Ken Rogers for production of SIVE660 virus for ELISA assays; Robert Wilson for assistance with rectal IgA assays; Jeffrey Americo for preparation of the MVA vaccine; Celia Labranche, William Rothwell, Corrine Rose and Cindi Emmerson for assistance with neutralization assays; Craig Auden, Jen Morgan, and Laura Hall and the genetics core of the New England Primate Research Center (NEPRC) for Trim5 genotyping; The Emory Center for AIDS Research (CFAR) virology core and Benton Lawson for viral load measurements, and Dr. Elizabeth Strobert and the Yerkes Research Center Veterinary Technicians for excellent animal care. We are indebted to Susan Reuland for administrative assistance.

\section{References}

1. Schneider J, Gilbert SC, Hannan CM, Degano P, Sheu EG, Plebanski M, et al. Induction of CD8+ T cells using heterologous prime-boost immunisation strategies. Immunological Reviews. 1999; 170:29-38. [PubMed: 10566139]

2. Robinson HL, Smith JM, Amara RR. AIDS Vaccines: Heterologous Prime/Boost Strategies for raising Protective T Cell Responses. AIDS Reviews. April-June; 2000 2(2):105-10.

3. Amara RR, Villinger F, Altman JD, Lydy SL, O’Neil SP, Staprans SI, et al. Control of a mucosal challenge and prevention of AIDS by a multiprotein DNA/MVA vaccine. Science. 2001 Apr 6; 292(5514):69-74. [PubMed: 11393868]

4. Liu J, O'Brien KL, Lynch DM, Simmons NL, La Porte A, Riggs AM, et al. Immune control of an SIV challenge by a T-cell-based vaccine in rhesus monkeys. Nature. 2009 Jan 1; 457(7225):87-91. [PubMed: 18997770]

5. Richmond JF, Lu S, Santoro JC, Weng J, Hu SL, Montefiori DC, et al. Studies of the neutralizing activity and avidity of anti-human immunodeficiency virus type 1 Env antibody elicited by DNA priming and protein boosting. Journal of Virology. 1998; 72(11):9092-100. [PubMed: 9765454]

6. Vaine M, Wang S, Hackett A, Arthos J, Lu S. Antibody responses elicited through homologous or heterologous prime-boost DNA and protein vaccinations differ in functional activity and avidity. Vaccine. Apr 9; 28(17):2999-3007. [PubMed: 20170767]

7. Amara RR, Villinger F, Staprans S, Altman JD, Montefiori D, Kozyr NL, et al. Different patterns of immune responses but similar control of a mucosal immunodeficiency virus challenge by MVA and DNA/MVA vaccines. Journal of Virology. 2002; 76:7625-31. [PubMed: 12097576]

8. Yeh WW, Jaru-Ampornpan P, Nevidomskyte D, Asmal M, Rao SS, Buzby AP, et al. Partial protection of Simian immunodeficiency virus (SIV)-infected rhesus monkeys against superinfection with a heterologous SIV isolate. J Virol. 2009 Mar; 83(6):2686-96. [PubMed: 19129440]

9. Reynolds MR, Weiler AM, Weisgrau KL, Piaskowski SM, Furlott JR, Weinfurter JT, et al. Macaques vaccinated with live-attenuated SIV control replication of heterologous virus. J Exp Med. 2008 Oct 27; 205(11):2537-50. [PubMed: 18838548]

10. Keele BF, Li H, Learn GH, Hraber P, Giorgi EE, Grayson T, et al. Low-dose rectal inoculation of rhesus macaques by SIV smE660 or SIVmac251 recapitulates human mucosal infection by HIV-1. J Exp Med. 2009 May 11; 206(5):1117-34. [PubMed: 19414559]

11. Van Rompay KK, Greenier JL, Cole KS, Earl P, Moss B, Steckbeck JD, et al. Immunization of newborn rhesus macaques with simian immunodeficiency virus (SIV) vaccines prolongs survival after oral challenge with virulent SIVmac251. J Virol. 2003 Jan; 77(1):179-90. [PubMed: 12477823]

12. Wyatt LS, Whitehead SS, Venanzi KA, Murphy BR, Moss B. Priming and boosting immunity to respiratory syncytial virus by recombinant replication-defective vaccinia virus MVA. Vaccine. 1999 Oct 14; 18(5-6):392-7. [PubMed: 10519927]

13. Wyatt LS, Belyakov IM, Earl PL, Berzofsky JA, Moss B. Enhanced cell surface expression, immunogenicity and genetic stability resulting from a spontaneous truncation of HIV Env expressed by a recombinant MVA. Virology. 2008 Mar 15; 372(2):260-72. [PubMed: 18048074]

14. Goepfert PA, Elizaga ML, Sato A, Qin L, Cardinali M, Hay CM, et al. Phase 1 safety and immunogenicity testing of DNA and recombinant modified vaccinia Ankara vaccines expressing HIV-1 virus-like particles. Journal of Infectious Disease. 2011 March; 203(1):610-9. 
15. Lai L, Kwa S, Kozlowski PA, Montefiori DC, Ferrari G, Johnson WE, et al. Prevention of Infection by a Granulocyte-Macrophage Colony-Stimualting Factor Co-Expressing DNA/ Modified Vaccinia Ankara Simian Immunodeficiency Virus Vaccine. The Journal of Infectious Diseases. 2011; 204:164-73. [PubMed: 21628671]

16. Kirmaier A, Wu F, Newman RM, Hall LR, Morgan JS, O'Connor S, et al. TRIM5 suppresses cross-species transmission of a primate immunodeficiency virus and selects for emergence of resistant variants in the new species. PLoS Biol. 2010; 8(8)

17. Lai L, Vodros D, Kozlowski PA, Montefiori DC, Wilson RL, Akerstrom VL, et al. GM-CSF DNA: an adjuvant for higher avidity $\operatorname{IgG}$, rectal $\mathrm{IgA}$, and increased protection against the acute phase of a SHIV-89.6P challenge by a DNA/MVA immunodeficiency virus vaccine. Virology. 2007 Dec 5; 369(1):153-67. [PubMed: 17698160]

18. Kozlowski PA, Lynch RM, Patterson RR, Cu-Uvin S, Flanigan TP, Neutra MR. Modified wick method using Weck-Cel sponges for collection of human rectal secretions and analysis of mucosal HIV antibody. J Acquir Immune Defic Syndr. 2000 Aug 1; 24(4):297-309. [PubMed: 11015145]

19. Bertley FM, Kozlowski PA, Wang SW, Chappelle J, Patel J, Sonuyi O, et al. Control of simian/ human immunodeficiency virus viremia and disease progression after IL-2-augmented DNAmodified vaccinia virus Ankara nasal vaccination in nonhuman primates. J Immunol. 2004 Mar 15; 172(6):3745-57. [PubMed: 15004179]

20. Montefiori, DC. Evaluating neutralizing antibodies against HIV, SIV and SHIV in a luciferase reporter gene assay. New York: John Wiley and Sons; 2004.

21. Pollara J, Hart L, Brewer F, Pickeral J, Packard BZ, Hoxie JA, et al. High-throughput quantitative analysis of HIV-1 and SIV-specific ADCC-mediating antibody responses. Cytometry A. 2011 Aug; 79(8):603-12. [PubMed: 21735545]

22. Jung T, Schauer U, Heusser C, Neumann C, Rieger C. Detection of intracellular cytokines by flow cytometry. J Immunol Methods. 1993 Feb 26; 159(1-2):197-207. [PubMed: 8445253]

23. Horton H, Thomas EP, Stucky JA, Frank I, Moodie Z, Huang Y, et al. Optimization and validation of an 8-color intracellular cytokine staining (ICS) assay to quantify antigen-specific T cells induced by vaccination. J Immunol Methods. 2007 May 31; 323(1):39-54. [PubMed: 17451739]

24. Hudgens MG, Gilbert PB. Assessing vaccine effects in repeated low-dose challenge experiments. Biometrics. 2009 Dec; 65(4):1223-32. [PubMed: 19397589]

25. Hudgens MG, Gilbert PB, Mascola JR, Wu CD, Barouch DH, Self SG. Power to detect the effects of HIV vaccination in repeated low-dose challenge experiments. J Infect Dis. 2009 Aug 15; 200(4):609-13. [PubMed: 19591571]

26. Seaman MS, Janes H, Hawkins N, Grandpre LE, Devoy C, Giri A, et al. Tiered categorization of a diverse panel of HIV-1 Env pseudoviruses for assessment of neutralizing antibodies. J Virol. 2010 Feb; 84(3):1439-52. [PubMed: 19939925]

27. Decker JM, Bibollet-Ruche F, Wei X, Wang S, Levy DN, Wang W, et al. Antigenic conservation and immunogenicity of the HIV coreceptor binding site. J Exp Med. 2005 May 2; 201(9):1407-19. [PubMed: 15867093]

28. Hel Z, Nacsa J, Tryniszewska E, Tsai WP, Parks RW, Montefiori DC, et al. Containment of simian immunodeficiency virus infection in vaccinated macaques: correlation with the magnitude of virus-specific pre- and postchallenge CD4+ and CD8+ T cell responses. J Immunol. 2002 Nov 1; 169(9):4778-87. [PubMed: 12391187]

29. Letvin NL, Rao SS, Montefiori DC, Seaman MS, Sun Y, Lim SY, et al. Immune and Genetic Correlates of Vaccine Protection Against Mucosal Infection by SIV in Monkeys. Sci Transl Med. 2011 May 4.3(81):81ra36.

30. Zhao J, Lai L, Amara RR, Montefiori DC, Villinger F, Chennareddi L, et al. Preclinical studies of human immunodeficiency virus/AIDS vaccines: inverse correlation between avidity of anti-Env antibodies and peak postchallenge viremia. J Virol. 2009 May; 83(9):4102-11. [PubMed: 19224993]

31. Pancera M, McLellan JS, Wu X, Zhu J, Changela A, Schmidt SD, et al. Crystal structure of PG16 and chimeric dissection with somatically related PG9: structure-function analysis of two quaternary-specific antibodies that effectively neutralize HIV-1. J Virol. 2010 Aug; 84(16):8098110. [PubMed: 20538861] 
32. Huber M, Trkola A. Humoral immunity to HIV-1: neutralization and beyond. J Intern Med. 2007 Jul; 262(1):5-25. [PubMed: 17598812]

33. Xiao P, Zhao J, Patterson LJ, Brocca-Cofano E, Venzon D, Kozlowski PA, et al. Multiple vaccineelicited nonneutralizing antienvelope antibody activities contribute to protective efficacy by reducing both acute and chronic viremia following simian/human immunodeficiency virus SHIV89.6P challenge in rhesus macaques. J Virol. 2010 Jul; 84(14):7161-73. [PubMed: 20444898]

34. Holl V, Peressin M, Decoville T, Schmidt S, Zolla-Pazner S, Aubertin AM, et al. Nonneutralizing antibodies are able to inhibit human immunodeficiency virus type 1 replication in macrophages and immature dendritic cells. J Virol. 2006 Jun; 80(12):6177-81. [PubMed: 16731957]

35. Edwards TG, Wyss S, Reeves JD, Zolla-Pazner S, Hoxie JA, Doms RW, et al. Truncation of the cytoplasmic domain induces exposure of conserved regions in the ectodomain of human immunodeficiency virus type 1 envelope protein. J Virol. 2002 Mar; 76(6):2683-91. [PubMed: 11861835]

36. Spies CP, Ritter GD Jr, Mulligan MJ, Compans RW. Truncation of the cytoplasmic domain of the simian immunodeficiency virus envelope glycoprotein alters the conformation of the external domain. J Virol. 1994 Feb; 68(2):585-91. [PubMed: 8289362]

37. Freed EO, Martin MA. Domains of the human immunodeficiency virus type 1 matrix and gp41 cytoplasmic tail required for envelope incorporation into virions. J Virol. 1996 Jan; 70(1):341-51. [PubMed: 8523546]

38. Zingler K, Littman DR. Truncation of the cytoplasmic domain of the simian immunodeficiency virus envelope glycoprotein increases env incorporation into particles and fusogenicity and infectivity. J Virol. 1993 May; 67(5):2824-31. [PubMed: 8474176]

39. Ritter GD Jr, Mulligan MJ, Lydy SL, Compans RW. Cell fusion activity of the simian immunodeficiency virus envelope protein is modulated by the intracytoplasmic domain. Virology. 1993 Nov; 197(1):255-64. [PubMed: 8212561]

40. Wyma DJ, Jiang J, Shi J, Zhou J, Lineberger JE, Miller MD, et al. Coupling of human immunodeficiency virus type 1 fusion to virion maturation: a novel role of the gp41 cytoplasmic tail. J Virol. 2004 Apr; 78(7):3429-35. [PubMed: 15016865]

41. Schwickert TA, Victora GD, Fooksman DR, Kamphorst AO, Mugnier MR, Gitlin AD, et al. A dynamic $\mathrm{T}$ cell-limited checkpoint regulates affinity-dependent B cell entry into the germinal center. J Exp Med. 2011 May 16.

42. Shih TA, Meffre E, Roederer M, Nussenzweig MC. Role of BCR affinity in T cell dependent antibody responses in vivo. Nat Immunol. 2002 Jun; 3(6):570-5. [PubMed: 12021782]

43. Salazar-Gonzalez JF, Salazar MG, Keele BF, Learn GH, Giorgi EE, Li H, et al. Genetic identity, biological phenotype, and evolutionary pathways of transmitted/founder viruses in acute and early HIV-1 infection. J Exp Med. 2009 Jun 8; 206(6):1273-89. [PubMed: 19487424] 


\section{Highlights}

Protective efficacy of MVA vaccine with and without a DNA prime

Different patterns of immune responses but similar protection

Potential role of form of Env in eliciting Ab with protective avidity 
A

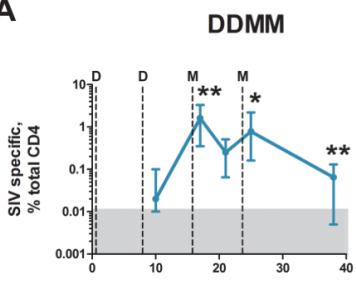

C

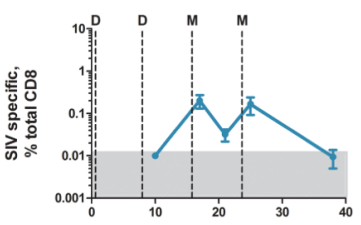

E

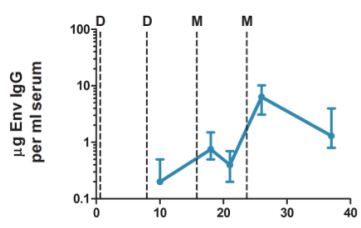

G

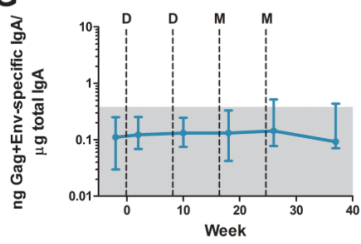

B

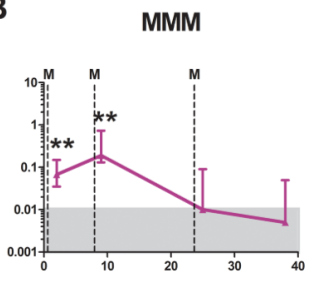

D

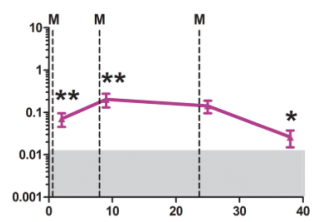

$\mathbf{F}$

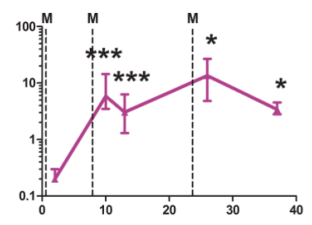

H

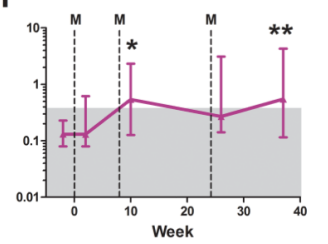

CD4

CD8

$\lg \mathbf{G}$

$\lg A$

Fig. 1. Temporal patterns of vaccine-elicited $\mathrm{T}$ cell and $\mathrm{Ab}$ responses

(A) and (B) IFN- $\gamma$ producing CD4 ICS responses to Gag and Env; (C) and (D) IFN- $\gamma$ producing CD8 ICS responses to Gag and Env; (E) and (F) Env-specific IgG in serum; (G) and $(\mathrm{H}) \mathrm{Gag}$,Pol plus Env-specific IgA in rectal secretions. Responses elicited by the DDMM regimen (turquoise) are in the left column; and those elicited by MMM (fuschia), in the right column. T cell responses were tested at 2 weeks following the $1^{\text {st }}$ inoculation, and then, at one week following each of the subsequent vaccine inoculations. Antibody responses were tested at 2 weeks following each inoculation. The memory response was tested at 13 weeks following the last inoculation (week 37). Data are medians with $25^{\text {th }}$ and $75^{\text {th }}$ quartiles indicated. Asterisks indicate responses that are significantly higher for one regimen than the other $\left(*, \mathrm{p}<0.05 ; *^{*}, \mathrm{p}<0.01 ; * * *, \mathrm{p}<0.001\right)$. For example in panels (A) and (B), CD4 T cell responses were significantly higher in the DDMM regimen at weeks 17, 25 and 37; and at weeks 2 and 9 in the MMM regimen. The grey boxes represent the backgrounds for detection of specific responses. 
A

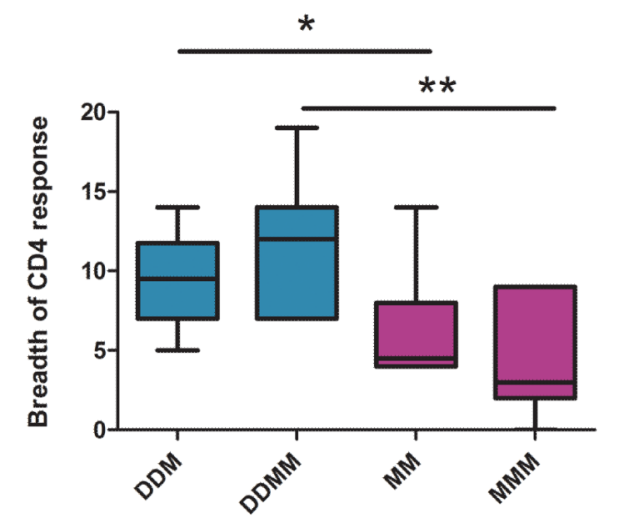

C

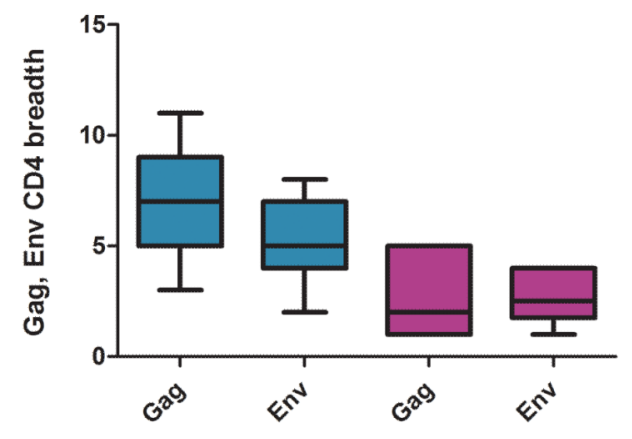

B

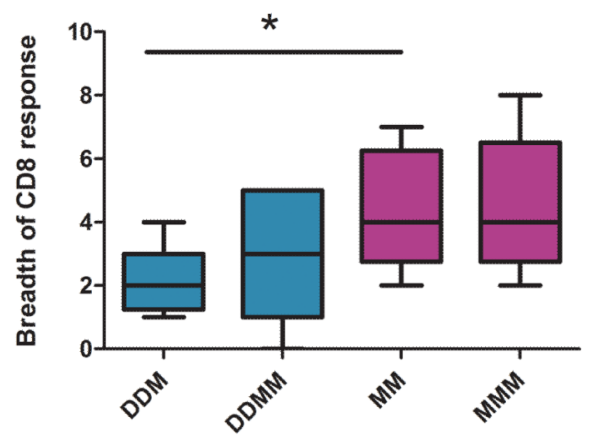

D

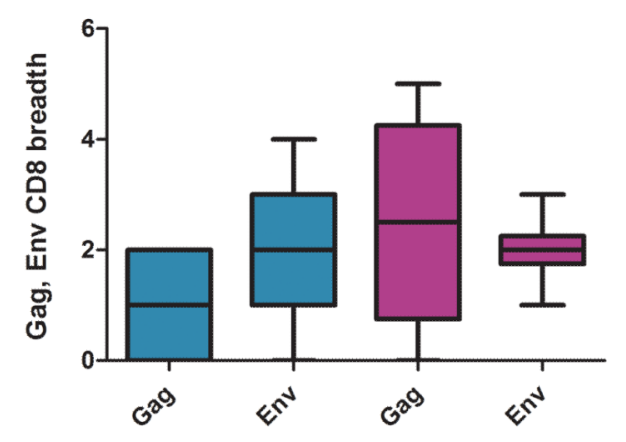

Fig. 2. Breadth of elicited CD4 and CD8 $\mathrm{T}$ cells

(A) and (B) Breadth of CD4 and CD8 T cell responses to 24 tested peptide pools; (C)and (D) Numbers of Gag and Env pools eliciting responses. CD4 T cell responses are in the left column; and CD8, in the right column. Responses for the DDMM regimen are in turquoise, and those for the MMM regimen, in fuchsia. The immunization regimen is indicated on the $\mathrm{X}$ axis using a D or M to indicate a DNA or MVA inoculation respectively. For example DDM indicates two DNA and one MVA inoculation. Boxplots present medians and interquartile ranges. $*$ is used to indicate $\mathrm{p}<0.05 ; * *, \mathrm{p}<0.01$; and $* * *, \mathrm{p}<0.001$. 
A

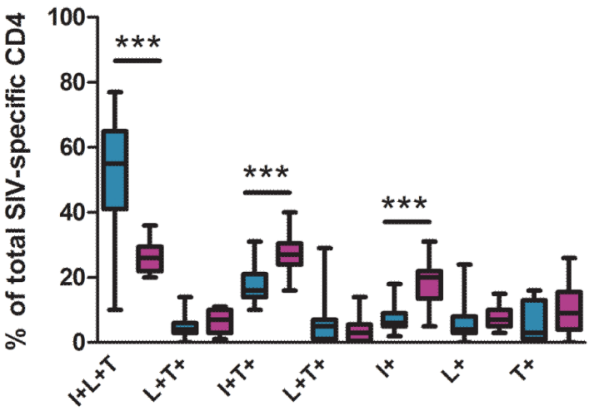

C

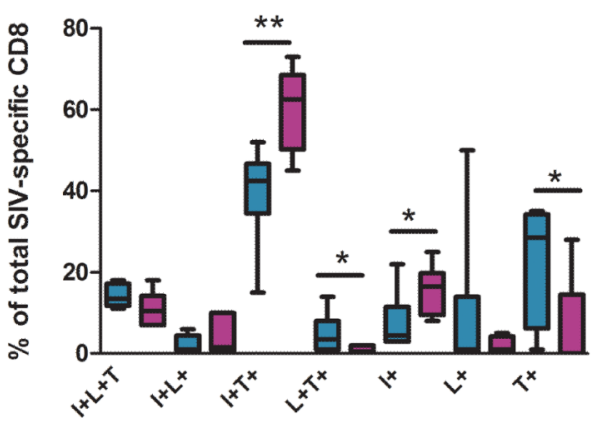

B Two Weeks post Infection

Fig. 3. Polyfunctionality of elicited CD4 and CD8 T cells at peak vaccine response and 2 weeks post infection

Intracellular cytokine staining was used to test for patterns of IFN- $\gamma$ (I), IL-2 (L) and TNF- $\alpha$ (T) production in peptide stimulated cells. Peak vaccine responses (left column) were analyzed for the DDMM regimen after the $1^{\text {st }}$ MVA boost; and for the MMM regimen, after the $2^{\text {nd }}$ MVA inoculation. The right column displays responses at 2 weeks post infection. Responses for the DDMM regimen are in turquoise; for the MMM regimen, in fuchsia and for unvaccinated controls, in white. Boxplots present medians and interquartile ranges. In (D), for the DDMM regimen, medians and interquartile range represent data for a sample size of 2 . $*$ is used to indicate $\mathrm{p}<0.05 ; * *, \mathrm{p}<0.01$; and $* * *, \mathrm{p}<0.001$. 
A

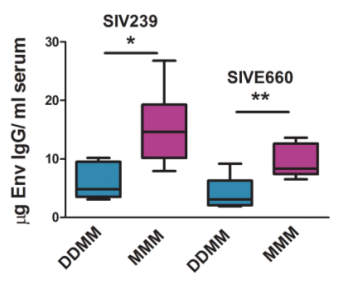

C

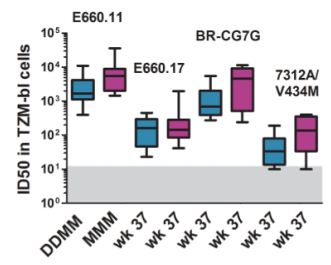

E

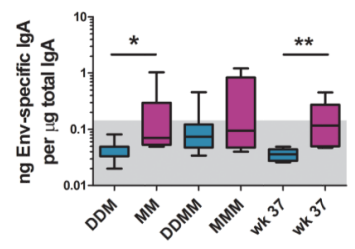

B

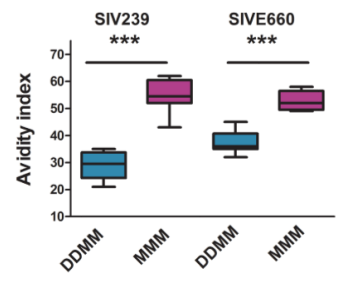

D

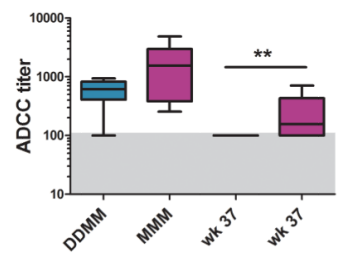

$\mathbf{F}$

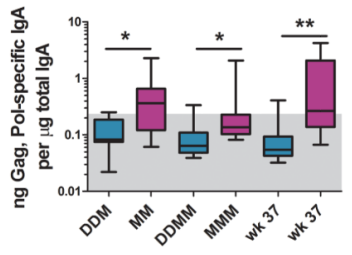

Fig. 4. Vaccine-elicited antibody responses

(A) Estimated titers of binding Ab for the SIV239 and SIVE660 Envs. (B) Avidity indices of the elicited Env specific Ab for the SIV239 and SIVE660 Envs. (C) Neutralizing titers of the elicited sera for tier 1 viruses. E660.11 and E660.17 are molecularly cloned Envs from the genetically complex challenge stock. SIVE660/CG7G is a molecular clone of a recent transmission [43]. 7312A/V33M is a HIV-2 virus mutated to test for the presence of neutralizing $\mathrm{Ab}$ for the CD4i site [27]. (D) ADCC activity of the elicited sera for SIV2339 gp120 coated CEM.NKR ${ }_{\text {CCR } 5}$ cells. (E) and (F) Env and Gag specific IgA in rectal secretions at different times in the trial. The DDMM group is depicted in turquoise and the MMM group, in fuchsia. The number of Ds and Ms indicate the number of immunizations. Wk 37 is a memory time point. The grey boxes in $(C)-(F)$ represent the level below which responses are not considered significant. $*$ is used to indicate $\mathrm{p}<0.05$; **, $\mathrm{p}<0.01$; and $* * *$, $\mathrm{p}<0.001$. 
A

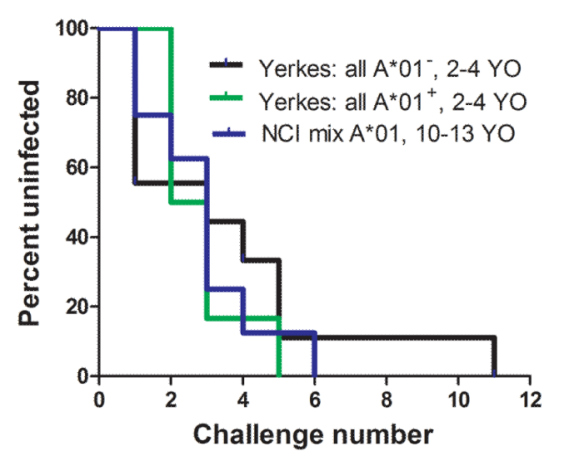

C

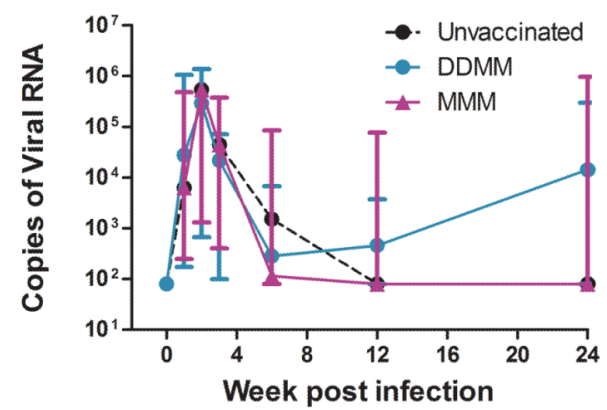

B

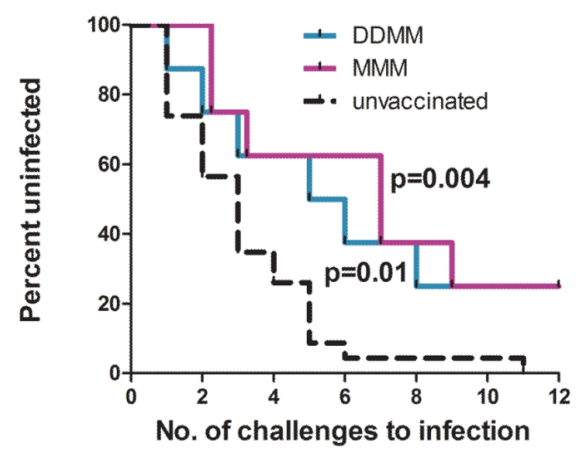

D

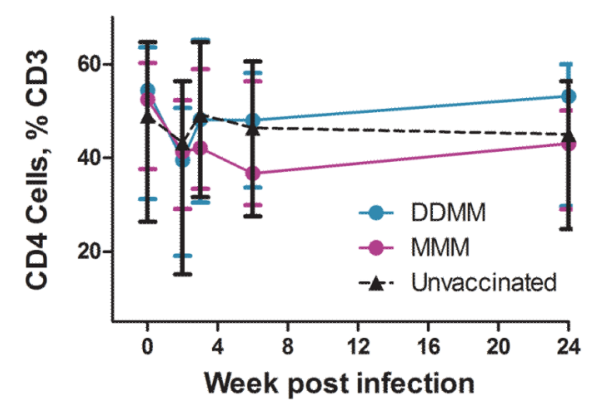

Fig. 5. Analyses for prevention of acquisition of a repeated rectal challenge and post infection levels of viral RNA, colorectal CD4 T cells, and Env specific Ab

(A) Results of 3 independent challenges using the SIVsmE660-Hirsch2000 stock under the same conditions. YO is year old. (B) Kaplan-Meier survival curves for vaccinated and pooled unvaccinated animals. The exact log-rank test indicates differences in the risk of infection for each of the two vaccine groups and the pooled control group, (C) Post challenge levels of viral RNA in animals that became infected. The day of infection is considered to be one week before virus was first detected in blood. (D) Post challenge levels of rectal CD4 T cells as a percent of total CD3 T cells. Data in (C) and (D) are medians with $25^{\text {th }}$ and $75^{\text {th }}$ percentiles indicated. Data for DDMM-vaccinated animals are in turquoise, those for MMM vaccinated animals in fuchsia, and those for unvaccinated animals in black. The grey box represents the level below which responses are not considered significant. 
A

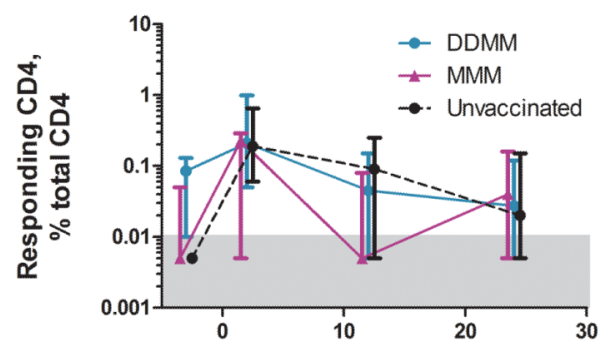

C

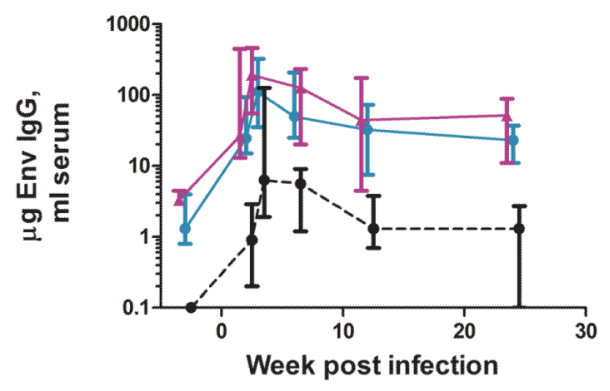

B

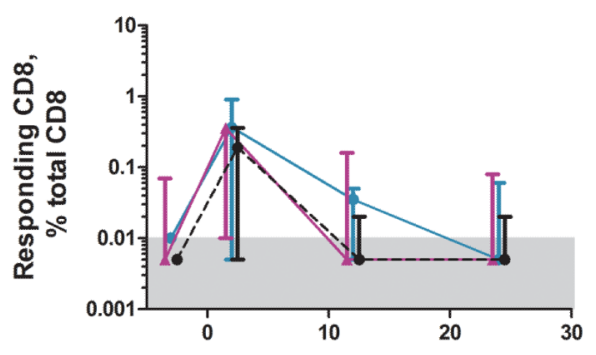

D

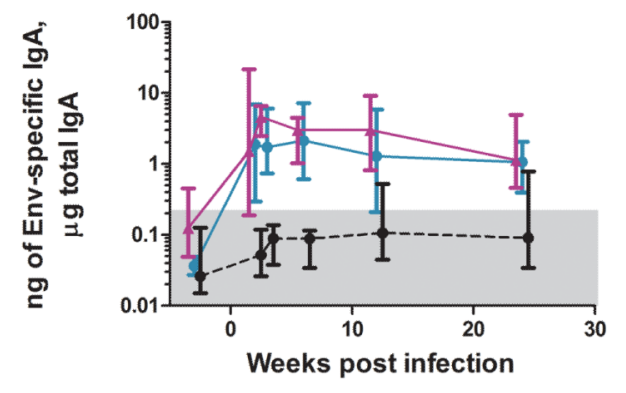

Fig. 6. Post infection immune responses

(A) Temporal IFN- $\gamma$ producing CD4 T cell responses, (B) Temporal IFN- $\gamma$ producing CD8 T cell responses, (C) Temporal Env-specific IgG responses in blood and (D) Temporal Envspecific IgA responses in rectal secretions. T cell responses are the sum of responses to Gag and Env. Responses in DDMM-vaccinated animals are in turquoise, those in MMM vaccinated animals, in fuchsia, and those for unvaccinated animals, in black. The plots of responses start with a week 48 prechallenge response. Responses have been displaced by $1 / 2$ week to better allow visualization of the different curves: DDMM is plotted at -0.5 weeks, MMM at the actual week and unvaccinated at +0.5 weeks. In (D), the grey box represents the level below which responses are not considered significant. * is used to indicate $\mathrm{p}<0.05$; $* *, \mathrm{p}<0.01 ;$ and $* * *, \mathrm{p}<0.001$. 
A

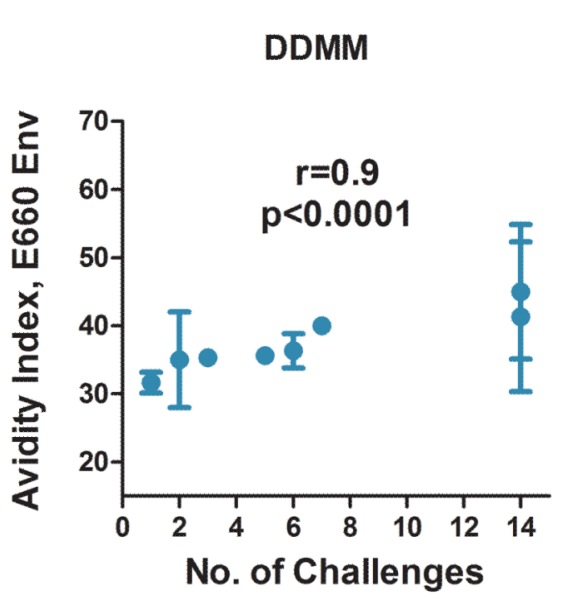

B

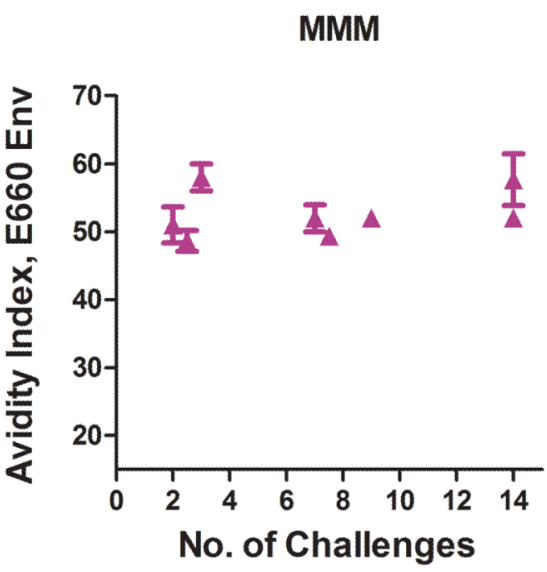

C

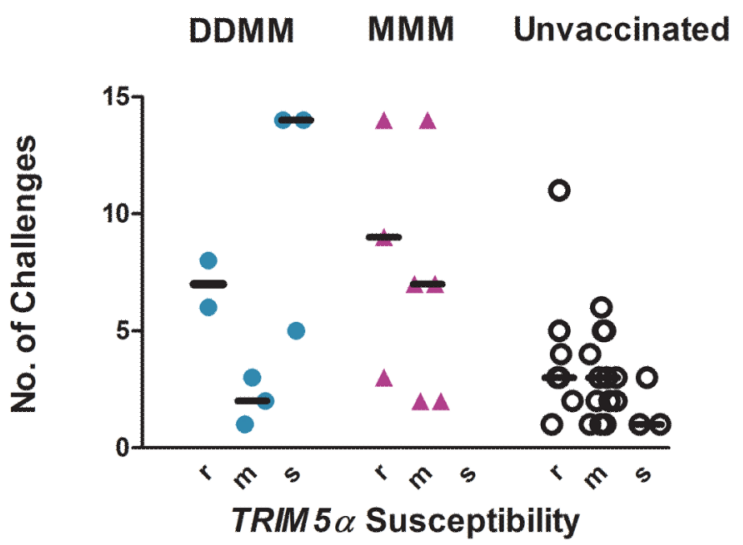

Fig. 7. Correlations

Correlation between number of challenges to infection and avidity of the vaccine-elicited $\mathrm{Ab}$ for the Env of the SIVE660 challenge in DDMM (A) but not MMM (B) vaccinated animals. Avidity measurements are for $\mathrm{Ab}$ collected at 2 weeks after the last immunization. (C) Lack of a correlation with prevention with TRIM5 $\alpha$ genotype. $\mathrm{r}$ designates a restrictive genotype; $\mathrm{m}$, a genotype that is heterozygous for restrictive (TRIM5 $\alpha^{\text {TFP }}$ or TRIM $5 \alpha^{C Y P A}$ ) and susceptible (TRIM5 $\left.\alpha^{Q}\right)$ genotypes, and s, a genotype that is homozygous for the susceptible genotype. The MMM group included no animals in the "s" group. DDMM animals are in turquoise, MMM vaccinated animals, in fuchsia; and unvaccinated animals, in black. Animals that did not become infected are plotted at 14 challenges. Data for individual animals can be found in supplementary Table 1 . 